\title{
Enteric nervous system $\alpha$-synuclein immunoreactivity in idiopathic REM sleep behavior disorder OPEN
}

Fabienne S. Sprenger, MD*

Nadia Stefanova, MD, PhD*

Ellen Gelpi, MD

Klaus Seppi, MD

Judith Navarro-Otano, MD

Felix Offner, MD

Dolores Vilas, MD

Francesc Valldeoriola, MD

Claustre Pont-Sunyer, MD

Iban Aldecoa, MD

Carles Gaig, MD

Angels Gines, MD

Miriam Cuatrecasas, MD, $\mathrm{PhD}$

Birgit Högl, MD

Birgit Frauscher, MD

Alex Iranzo, MD

Gregor K. Wenning, MD, $\mathrm{PhD}$

Wolfgang Vogel, MD

Eduardo Tolosa, MD

Werner Poewe, MD

Correspondence to Dr. Poewe:

Werner.Poewe@i-med.ac.at

Editorial, page 1732

\section{ABSTRACT}

Objective: To investigate the expression of $\alpha$-synuclein in colonic biopsies of patients with idiopathic REM sleep behavior disorder (iRBD) and address if $\alpha$-synuclein immunostaining of tissue obtained via colonic biopsies holds promise as a diagnostic biomarker for prodromal Parkinson disease (PD).

Methods: Patients with iRBD, patients with PD, and healthy controls were prospectively recruited to undergo colonic biopsies for comparison of $\alpha$-synuclein immunoreactivity patterns between the groups by using 2 different antibodies.

Results: There was no difference in colonic mucosal and submucosal immunostaining between groups using the $15 \mathrm{G} 7 \alpha$-synuclein antibody, which was found in almost all participants enrolled in this study. By contrast, immunostaining for serine 129-phosphorylated $\alpha$-synuclein (pSyn) in submucosal nerve fibers or ganglia was found in none of 14 controls but was observed in 4 of 17 participants with iRBD and 1 out of 19 patients with PD.

Conclusions: The present findings of pSyn immunostaining of colonic biopsies in a substantial proportion of iRBD participants raise the possibility that this tissue marker may be a suitable candidate to study further as a prodromal PD marker in at-risk cohorts. Neurology ${ }^{\circledR}$ 2015;85:1761-1768

\section{GLOSSARY}

ANS = autonomic nervous system; ENS = enteric nervous system; HC = healthy control; iRBD = idiopathic REM sleep behavior disorder; MDS-UPDRS = Movement Disorder Society-Unified Parkinson's Disease Rating Scale; PD = Parkinson disease; $\mathbf{p S y n}=$ phosphorylated $\alpha$-synuclein; UPSIT = University of Pennsylvania Smell Identification Test.

Intraneuronal Lewy bodies and Lewy neurites consisting of aggregated $\alpha$-synuclein are the hallmark of brain pathology in Parkinson disease (PD). Lewy body pathology in PD is not confined to the CNS and involvement of the peripheral autonomic nervous system (ANS) has already been observed more than 50 years ago. ${ }^{1}$ Recently there has been renewed emphasis on the peripheral ANS as a site of $\alpha$-synuclein pathology in PD supported by findings of Lewy body pathology or positive $\alpha$-synuclein immunohistochemistry in the enteric or cardiosympathetic nervous system of patients with PD., In addition, several studies found positive $\alpha$-synuclein staining in colonic biopsy tissue obtained from participants with PD. ${ }^{4-7}$

Positive immunostaining for $\alpha$-synuclein was also reported in submucosal tissue of archival material from colonic biopsies in 3 cases of PD where colonoscopies had been performed for routine purposes several years prior to onset of PD. ${ }^{8}$ These findings raise the question whether $\alpha$-synuclein immunostaining of tissue obtained via colonic biopsies holds promise as a diagnostic biomarker for PD even prior to the occurrence of diagnostic clinical motor features.

The present study was performed to investigate this question by obtaining colonic biopsies from participants with idiopathic REM sleep behavior disorder (iRBD), a condition that may

\footnotetext{
*These authors contributed equally to this work.

From the Departments of Neurology (F.S.S., N.S., K.S., B.H., B.F., G.K.W., W.P.) and Gastroenterology (W.V.), Medical University of Innsbruck, Austria; Neurological Tissue Bank of the Biobanc-Hospital Clinic de Barcelona (E.G.), IDIBAPS; the Departments of Neurology (J.N.-O., D.V., F.V., C.P.-S., C.G., A.I., E.T.), Pathology (I.A., M.C.), and Gastroenterology (A.G.), Hospital Clinic de Barcelona, Spain; and the Institute of Pathology (F.O.), Academic Teaching Hospital, Feldkirch, Austria.

Go to Neurology.org for full disclosures. Funding information and disclosures deemed relevant by the authors, if any, are provided at the end of the article. The Article Processing Charge was paid by grant F4414 of the Austrian Science Fund (FWF).

This is an open access article distributed under the terms of the Creative Commons Attribution-NonCommercial-NoDerivatives License 4.0 (CC BY-NC-ND), which permits downloading and sharing the work provided it is properly cited. The work cannot be changed in any way or used commercially.
} 
represent prodromal PD in up to $80 \%$ of patients according to longitudinal studies. ${ }^{9-12}$ $\alpha$-Synuclein immunostaining in mucosal and submucosal biopsy material was compared to findings in healthy controls (HCs) and patients with clinically established PD.

METHODS Participants. Patients with polysomnographic confirmed RBD but no clinical signs of parkinsonism, cognitive dysfunction, or other clinical evidence for a neurodegenerative disease (iRBD), patients with a diagnosis of PD according to the UK Parkinson's Disease Society Brain Bank, ${ }^{13}$ as well as HCs with no evidence of neurologic disease or dream-enacting behaviors were prospectively and consecutively enrolled in this study to obtain colonic biopsies by flexible sigmoidoscopy or colonoscopy. Control participants were recruited from the gastroenterology service of the 2 hospital sites and indications for colonoscopy included routine preventive cancer screening, follow-up of colonic adenomas, as well as workup for diarrhea.

Standard protocol approvals, registrations, and patient consents. Each participating site (Department of Neurology, Medical University of Innsbruck, and Department of Neurology and Pathology, Hospital Clinic de Barcelona) received approval from an ethical standards committee on human experimentation before study initiation and obtained written informed consent for research from all individuals participating in the study.

Clinical assessments. Clinical assessments included the Movement Disorder Society-Unified Parkinson's Disease Rating Scale (MDS-UPDRS) parts I to III to assess motor and nonmotor symptoms of parkinsonism. ${ }^{14}$ Smell function was assessed using Sniffin' Sticks (identification, 16-item; Innsbruck site) or the University of Pennsylvania Smell Identification Test (UPSIT; Barcelona site).

Colonic biopsies. Flexible sigmoidoscopy was used in patients recruited at the Innsbruck site and performed according to the standard procedure of the Gastroenterology Department of Innsbruck Medical University Hospital. Biopsies were taken in the rectum or sigma using standard biopsy forceps without needles. The Barcelona site used standard colonoscopies and 3 to 5 biopsies were obtained from ascending, transverse, descending, and sigmoid colon using the standard biopsy forceps.

Tissue preparation, immunohistochemistry, and image analysis. Paraffin-embedded $10 \%$ formalin-fixed biopsies were cut at $4 \mu \mathrm{m}$. Sections were deparaffinized according to a standard protocol. Antigen retrieval was performed with $10 \mathrm{mM}$ sodium acetate $\mathrm{pH} 6$, endogenous peroxidase was blocked with $3 \% \mathrm{H}_{2} \mathrm{O}_{2}$, and nonspecific binding was blocked by incubation with $10 \%$ normal goat serum. Immunohistochemical staining was performed by incubation with a primary antibody at $4^{\circ} \mathrm{C}$ overnight followed by suitable biotinylated immunoglobulin G (Vector Laboratories, Burlingame, CA) and avidin-biotin peroxidase Elite Kit (Vector Laboratories). Immunoreactivity was visualized by $3,3^{\prime}$ diaminobenzidine. The primary antibodies used in this study were rat anti-human $\alpha$-synuclein (15G7, Enzo Life Sciences, Lörrach, Germany) and mouse anti-phosphorylated $\alpha$-synuclein (pSyn, Wako, Neuss, Germany). Image analysis was performed with the aid of a Nikon (Tokyo, Japan) Eclipse E800 microscope provided with a Nikon digital camera DXM 1200. Ratings were performed by 2 independent observers (N.S. and E.G.), blinded to the diagnosis of the cases. Mucosa and submucosa were rated negative (0) or positive (1) for labeled neuronal cell bodies, nerve fibers, and non-neural structures (macrophages).

Immunofluorescence was performed to identify the colocalization of human $\alpha$-synuclein with the neuronal marker anti$\mathrm{HuC} / \mathrm{HuD}$ (Molecular Probes, Leiden, the Netherlands). The incubation with the primary antibodies was followed by the labeling of the suitable secondary Alexa 594- or Alexa 488-conjugated antibodies. DAPI nucleic acid stain (Molecular Probes, Eugene, $\mathrm{OR}$ ) was used for counterstaining. Fluorescence image analysis was performed under DMI 4000B Leica (Wetzlar, Germany) microscope provided with the Application Suite V3.1 and a Digital Fire Wire Color Camera DFC300 FX.

Statistical analyses. The primary outcome measure of this study was the overall frequency of any positive nerve cell or fiber $\alpha$-synuclein immunostaining. Group comparisons for categorical variables were performed using $\chi^{2}$ tests with post hoc Fisher exact tests. Parametric or nonparametric tests were used for group

\section{Table 1 Demographic and clinical data of patients with PD, patients with iRBD, and HCs}

\begin{tabular}{|c|c|c|c|c|c|c|c|}
\hline & $\mathrm{HC}(\mathrm{n}=22)$ & $\mathrm{PD}(\mathrm{n}=24)$ & iRBD $(n=20)$ & p Value, all & PD vs HC & PD vs iRBD & RBD vs HC \\
\hline Age, $y$, mean $\pm S D$ & $65.7 \pm 9.46$ & $64.39 \pm 9.14$ & $70.28 \pm 5.29$ & $0.059^{a}$ & & & \\
\hline Disease duration, $y$, mean $\pm S D$ & NA & $8.99 \pm 5.43$ & $7.82 \pm 6.90$ & $0.542^{a}$ & & & \\
\hline Female, \% (n) & $27.27(6)$ & $33.3(8)$ & $15.0(3)$ & $0.376^{b}$ & & & \\
\hline Sniffin' Sticks, mean \pm SD & $12.33 \pm 3.00^{c}$ & $7.70 \pm 2.50^{c}$ & $8.40 \pm 3.95^{c}$ & $0.017^{c, d}$ & $0.003^{c}$ & 0.853 & 0.035 \\
\hline UPSIT, mean \pm SD & $28.67 \pm 5.79^{c}$ & $17.67 \pm 6.30^{c}$ & $23.11 \pm 7.31^{c}$ & $0.003^{c, d}$ & $0.001^{c}$ & 0.069 & 0.031 \\
\hline MDS-UPDRS, part I, mean \pm SD & $4.89 \pm 4.64^{c}$ & $9.25 \pm 5.71^{c}$ & $7.65 \pm 4.75^{c}$ & $0.051^{d}$ & & & \\
\hline MDS-UPDRS, part II, mean \pm SD & $1.95 \pm 4.47^{c}$ & $10.79 \pm 5.75^{\mathrm{c}}$ & $2.9 \pm 2.86^{c}$ & $<0.001^{c, d}$ & $<0.001^{c}$ & $<0.001^{c}$ & 0.727 \\
\hline MDS-UPDRS, part III, mean \pm SD & $2.6 \pm 3.27^{c}$ & $22.63 \pm 12.85^{c}$ & $5.2 \pm 6.16^{c}$ & $<0.001^{\mathrm{c}, \mathrm{d}}$ & $<0.001^{\mathrm{c}}$ & $<0.001^{c}$ & 0.444 \\
\hline
\end{tabular}

Abbreviations: $\mathrm{HC}=$ healthy control; $\mathrm{RBBD}=$ idiopathic REM sleep behavior disorder; MDS-UPDRS = Movement Disorder Society-Unified Parkinson's Disease Rating Scale; NA = not applicable; PD = Parkinson disease; UPSIT = University of Pennsylvania Smell Identification Test.

${ }^{\text {a }}$ One-way analysis of variance.

${ }^{b}$ Chi-square tests with post hoc Fisher exact tests corrected for multiple comparisons setting the $p$ values at $p<0.0167(0.05 / 3)$.

c Significant.

${ }^{d}$ Kruskal-Wallis with post hoc Wilcoxon-Mann-Whitney $U$ tests corrected for multiple comparisons setting the $p$ values at $p<0.0167(0.05 / 3)$. 
comparisons depending on the scale type of the variables. Between-group comparisons among iRBD, PD, and controls were performed with a 1-way analysis of variance with post hoc Bonferroni correction for age and disease duration; with the Kruskal-Wallis 1-way analysis of variance followed by post hoc Mann-Whitney $U$ tests for the Hoehn \& Yahr scale, UPSIT, Sniffin' Sticks, and MDS-UPDRS as well as with the $\chi^{2}$ test for sex distribution. Post hoc Mann-Whitney $U$ tests and Fisher exact tests were corrected for multiple comparisons $(p<$ $0.05 / 3=0.017)$ and performed if the $p$ value of the KruskalWallis 1 -way analysis of variance or $\chi^{2}$ test was $\leq 0.100$. The significance level was set at $p<0.05$. Data were tabulated and analyzed using SPSS 21.0 for Windows (SPSS, Inc., Chicago, IL).

RESULTS Clinical assessments. A total of 20 patients with iRBD, 24 patients with PD, and $22 \mathrm{HCs}$ consented to participate and were enrolled in this study.

As expected, patients with PD had significantly higher scores on the MDS-UPDRS parts I, II, and III as compared to both patients with iRBD and HCs. Both patients with PD and patients with iRBD had significantly lower UPSIT and Sniffin' Sticks scores as compared to HCs (table 1).

$\alpha$-Synuclein expression in colonic biopsies detected with 15G7 antibody. Sufficient biopsy material for the histologic analysis was available for all participants.
While all samples contained adequate mucosal tissue for $\alpha$-synuclein and pSyn immunostaining analysis, submucosal tissue was not invariably included in the biopsy sections such that only a subset of participants (16 HCs, 21 PD, and 17 iRBD) could be analyzed.

Localization and patterns of $15 G 7 \alpha$-synuclein immunostaining. Immunostaining for $\alpha$-synuclein (15G7 antibody) was identified in both the mucosal and submucosal parts of biopsies in almost all iRBD cases and HCs and in all patients with PD. Mucosal staining had predominantly nerve fiber-like distribution (figure 1, A and C). Similar to previous reports, ${ }^{15}$ in some cases immunopositive mucosal macrophages were present as well, often forming clusters in the lamina propria or at the border between lamina propria and muscularis mucosae (figure 1B).

In the submucosa, both nerve fibers and submucosal ganglionic cells were immunopositive for the 15G7 antibody (figure 1, D-G). In submucosal ganglia, 3 different patterns of distribution of the immunopositive material were observed: diffuse cytoplasmic staining (figure 1E), distinct punctate (figure $1 \mathrm{~F}$ ), or coarse aggregate-like patterns (figure $1 \mathrm{G}$ ). The neuronal localization of $\alpha$-synuclein was confirmed by costaining with the neuronal marker $\mathrm{HuC} / \mathrm{HuD}$ (figure 2).
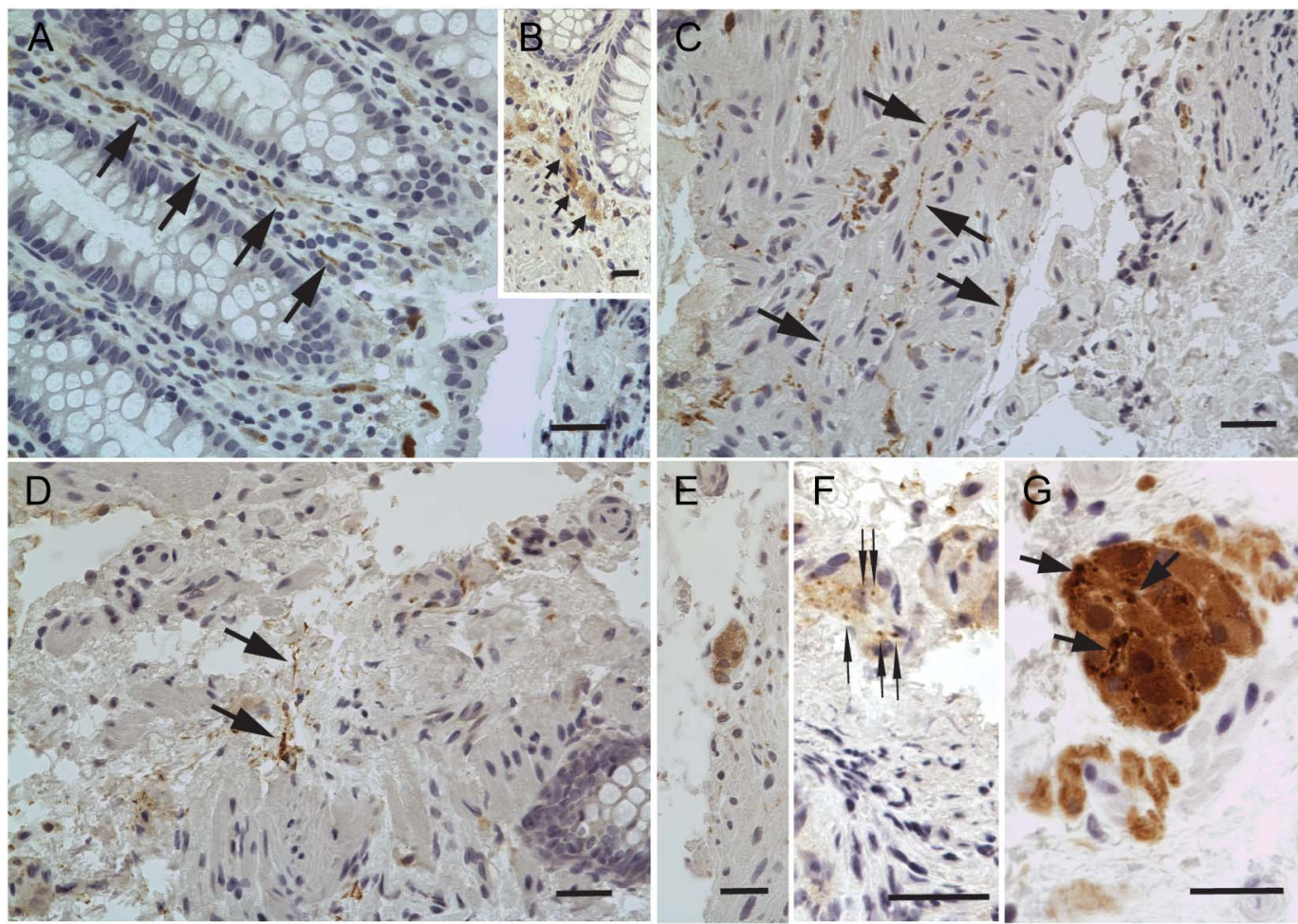

Total $\alpha$-synuclein expression in colonic biopsies detected with the $15 \mathrm{G} 7$ antibody. (A) $\alpha$-Synuclein-positive nerve fibers (arrows) were identified in lamina propria of the mucosa. (B) $\alpha$-Synuclein expression was detected with the 15G7 antibody in mucosal macrophages (arrows) in the lamina propria at the border to muscularis mucosae. (C) Immunopositive nerve fibers (arrows) were also commonly seen in muscularis mucosae. (D) $\alpha$-Synuclein-positive nerve fibers (arrows) were detected in the submucosa of colonic biopsies. Submucosal ganglionic nerve cells showed variable pattern of $\alpha$-synuclein immunostaining in the cytoplasm: (E) diffuse; (F) distinct punctate (arrows); or (G) coarse aggregate-like (arrows). Scale bars, $20 \mu \mathrm{m}$. 

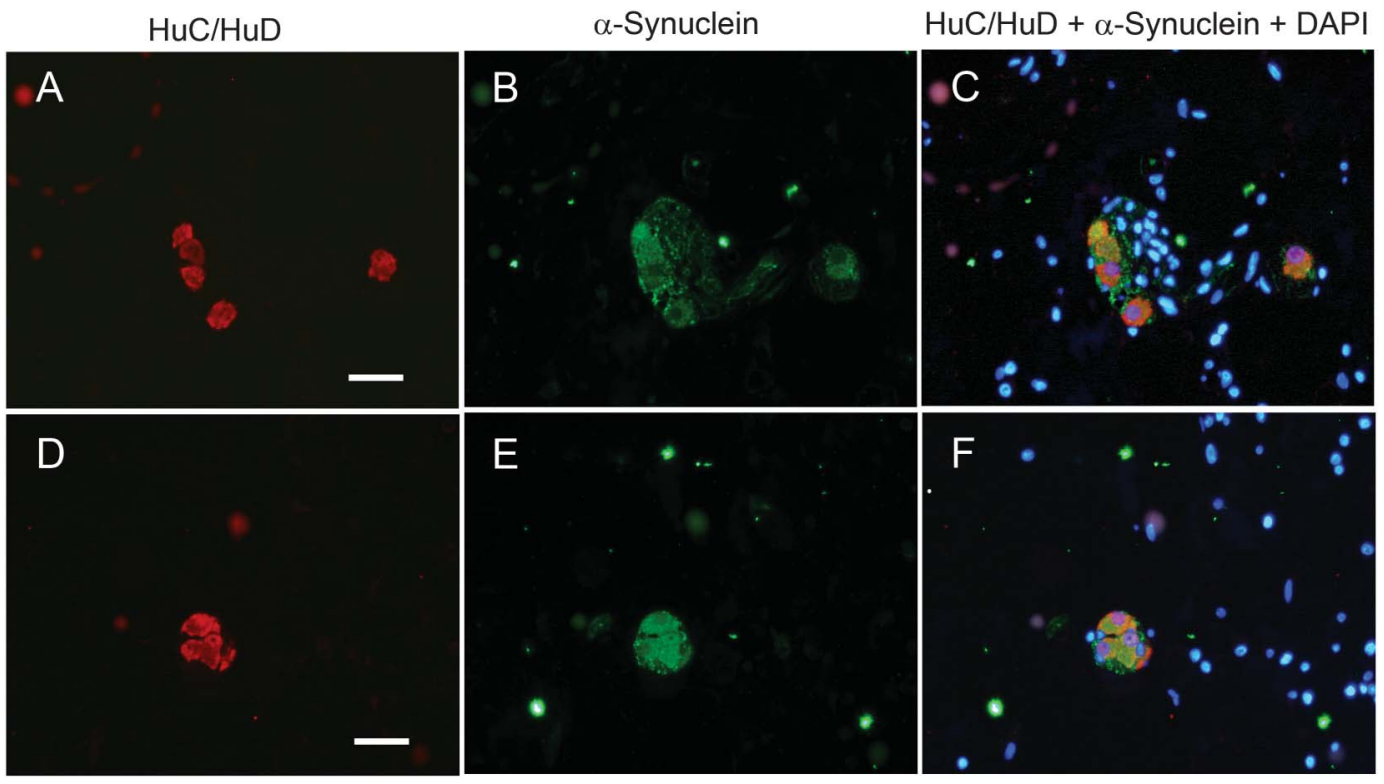

Submucosal ganglionic nerve cells coexpress the neuronal marker HuC/HuD (red, A, D) and $\alpha$-Synuclein identified by $15 \mathrm{G} 7$ antibody (green, B, E), as proven by the merge of the labeling in $\mathrm{C}$ and $\mathrm{F}$, with blue representing DAPI nuclear counterstaining. Scale bars, $20 \mu \mathrm{m}$.

Prevalence of positive $15 G 7 \alpha$-synuclein immunostaining. A total of 19 of 20 patients with iRBD (95\%), 21 of 22 HCs (95\%), and all 24 patients with PD (100\%) had $\alpha$-synuclein immunopositive nerve fibers in the mucosal layer. Immunopositive mucosal macrophages were also identified in biopsies taken from participants of all groups (HCs 55\% [12 of 22], PD $42 \%$ [10 of 24], and RBD $40 \%$ [8 of 20]).

15G7 immunopositive nerve fibers or ganglia were also detectable in the submucosa in all participants irrespective of diagnostic group: $100 \%$ of the $\mathrm{iRBD}$ participants, PD participants, and HCs had this finding in at least one biopsy sample. 15G7-positive submucosal nerve fibers were observed more frequently in 14 of 16 patients with iRBD (88\%) and 13 of 21 patients with $\mathrm{PD}(62 \%)$ compared to 8 of 16 HCs (50\%), but there were no differences in 15G7-immunopositive ganglia in the submucosa, which were found in $88 \%$ of the $\mathrm{HC}$ biopsies, in $94 \%$ of the iRBD biopsies, and in $100 \%$ of the PD biopsies. No differences among iRBD cases, patients with PD, and HCs in the mucosal and submucosal 15G7 immunostaining were identified when the analysis was performed separately for rostral and caudal levels of colonic biopsies (table 2).

Phosphorylated $\alpha$-synuclein expression in PD, iRBD, and HC. We did not detect positive mucosal immunostaining for phosphorylated $\alpha$-synuclein (pSyn) in any biopsy taken from any group at any level of the colon (rostral: ascending, transverse, descending colon, or caudal: sigma/rectum). By contrast, pSyn immunostaining (figure 3) was identified in the submucosa of colonic biopsies of 4 (out of 17) patients with iRBD with positive ganglionic cell bodies in 3 and positive fibers in all 4 cases. Of these 4 iRBD cases, 1 had pSyn-positive immunostaining in the caudal colon, in 2 cases immunopositivity was identified in rostral levels only, and 1 iRBD case was positive for $\mathrm{pSyn}$ at both rostral and caudal levels. A single PD case (out of 19 suitable for evaluation at the level of submucosa) had pSynpositive fibers and ganglionic cell bodies in the submucosa of the sigmoid colon, but none of the more rostral biopsies showed pSyn immunopositive staining in any participant with PD. None of the biopsies obtained from HCs stained for pSyn in the submucosa, and there was a significant group effect for rostral submucosal staining. However, post hoc testing between groups failed to reach statistical significance (table 2).

Of the 4 pSyn-immunopositive patients with iRBD, 1 had hyposmia, 2 were normosmic, and 1 was excluded from testing due to chronic rhinitis, as compared to 9 hyposmic participants among the biopsy-negative iRBD cases. Constipation as defined as a score of at least 2 in the MDS-UPDRS (I) item 11 was present in 1 of the 4 biopsy-positive cases as compared to 4 of the biopsy-negative cases.

DISCUSSION This study assesses $\alpha$-synuclein immunohistochemistry in colonic biopsies obtained from patients with iRBD and compares findings to 


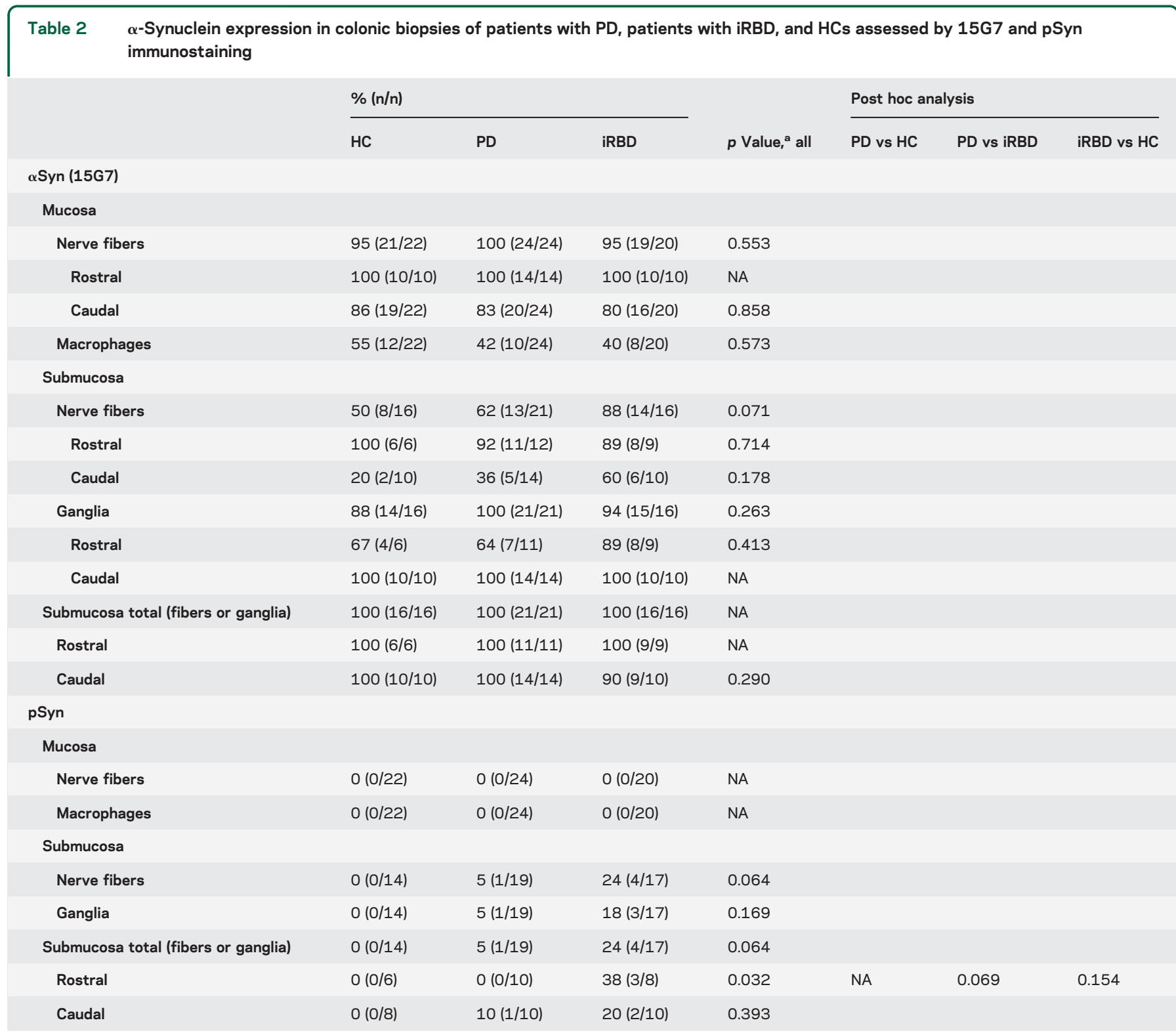

Abbreviations: HC = healthy controls; iRBD = idiopathic REM sleep behavior disorder; NA = not applicable; PD = Parkinson disease.

${ }^{a}$ Chi-square tests with post hoc Fisher exact tests corrected for multiple comparisons setting the $p$ values at $p<0.0167(0.05 / 3)$.

those in HCs and patients with PD. Using the 15G7 antibody, which binds to amino acids 116-131 of native $\alpha$-synuclein regardless of its aggregation status, there was positive immunostaining of nerve fibers and ganglia in the colonic mucosa and submucosa for almost all participants in all groups. This argues against a role of enteric nervous system (ENS) immunostaining with this antibody as a diagnostic biomarker for PD or its prodromal stages. Our findings are thus in contrast to a previous report investigating $\alpha$-synuclein immunoreactivity in biopsies of the distal sigmoid colon in 10 patients with early untreated PD compared to 23 controls using the antibody LB509, which binds to amino acids 115122 of native $\alpha$-synuclein. ${ }^{6}$ In that report, immunostaining of nerve fibers of the colonic submucosa was observed in all PD biopsies but in none of the controls. However, later studies applying different $\alpha$-synuclein antibodies have reported contrasting findings and provided evidence that $\alpha$-synuclein is a normal constituent of the ENS and thus present both in HCs as well as participants with PD. ${ }^{7,16}$ Indeed, a detailed morphologic study on colonic whole wall resections from patients without $\mathrm{PD}$ with intestinal tumors also found positive immunostaining for $\alpha$-synuclein in all samples. ${ }^{16}$ A recent study used both conventional immunohistochemistry and aggregate-specific staining via PET blots to possibly identify diseasespecific patterns in PD but also failed to detect differences in the number of participants with positive staining of colonic biopsies between participants with PD and HCs. ${ }^{17}$ Taken together, 


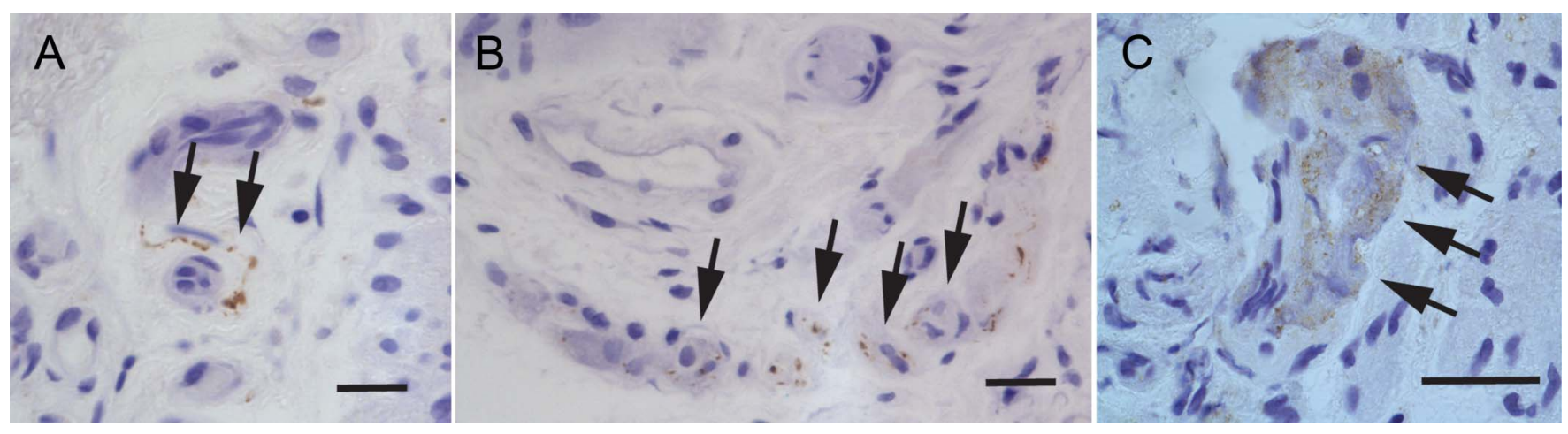

Phosphorylated $\alpha$-synuclein (pSyn) submucosal staining patterns in colonic biopsies. (A) pSyn-immunopositive nerve fiber in the submucosa (arrows). (B) pSyn-positive aggregates in the cytoplasm of submucosal ganglionic nerve cells (arrows). (C) Diffuse pSyn cytoplasmic immunoreactivity in submucosal ganglia (arrows). Scale bars, $20 \mu \mathrm{m}$.

immunohistochemical studies on colonic biopsies using antibodies binding to native $\alpha$-synuclein, including the present series, do not support a role of this approach as a useful PD biomarker.

Intriguingly, assessment of $\alpha$-synuclein immunohistochemistry with the LB509 antibody in archival colonic biopsy material obtained in 3 patients with PD 2-5 years before diagnosis again found immunostaining in all of them ${ }^{8}$ and suggested that $\alpha$-synuclein immunohistochemistry in colonic mucosa and submucosa could be a specific marker for PD even in prediagnostic cases. This notion also gained support from a postmortem study that reported that $\alpha$-synuclein identified by the antibody LB509 was present both in patients with $\mathrm{PD}$ and in pathologically defined cases of incidental Lewy body disease but not in controls. ${ }^{18}$ In this study, we investigated participants with $\mathrm{RBD}$ as a surrogate for prodromal PD and using the 15G7 antibody all showed immunopositive fibers and nerve cells at the mucosal or submucosal level. However, this was not different from HCs. By contrast, pSyn immunostaining, which might be more specific for PD due to the hyperphosphorylation of $\alpha$-synuclein associated with the disease, ${ }^{19,20}$ was seen in 4 of 17 participants with iRBD at the submucosal level and was not detected in any of the controls. Therefore, the present findings raise the possibility that ENS pSyn immunohistochemistry could help to identify those participants with iRBD who will go on to develop clinical PD and in whom RBD could be considered a prodromal manifestation of the disease. Moreover, it would be broadly consistent with hypotheses postulating the ENS as an initiating site of $\alpha$-synuclein pathology with subsequent transsynaptic spread via the vagal nerve to the caudal brainstem and eventually more rostrally to the midbrain and cortical areas. ${ }^{2,21,22}$ However, within the constraints of increased thresholds for statistical significance due to multiple testing among 3 groups, the present finding of a difference in the number of immunopositive cases between iRBD and the other groups is not statistically significant. Also, if pSyn immunopositivity in the ENS were to indicate prodromal $\mathrm{PD}$, it is difficult to understand why only a single patient with $P D$ in this series was positive for this marker. A speculative explanation could be that after initiation of $\alpha$-synuclein pathology in the ENS, rostral transport or transmission might be associated with clearance of aggregated $\alpha$-synuclein from enteric neurons. Indeed, centripetal distribution of $\alpha$-synuclein from distal fibers in the heart to neuronal somata in paravertebral sympathetic fibers has been reported. ${ }^{23}$ Alternatively, as pathology progresses from prodromal PD to clinically established disease, there might be loss of enteric neurons and nerve fibers, thus reducing immunopositivity, but this has not been investigated quantitatively in PD to date. Nevertheless, observations in skin biopsies support the hypothesis of peripheral nerve fiber loss as an intrinsic feature of PD. ${ }^{24}$

It has to be acknowledged, however, that previous studies using antibodies against pSyn in colonic biopsies of PD have yielded conflicting results. Similar to this series, pSyn pathology in form of positively stained Lewy neurites was reported in none of the controls but-differently from our resultsin $72 \%$ of the patients with PD. ${ }^{5}$ Another report of a microdissection study of colonic mucosa from biopsies also identified pSyn-positive Lewy neurites in the submucosal plexus and mucosa $(14 \%$ and $33 \%$, respectively) of the sigmoid and ascending colon in a proportion of patients with PD but not in HCs. ${ }^{25}$ In contrast, however, others found positive immunostaining for $\mathrm{pSyn}$ in the majority of patients with early and late PD and HCs. ${ }^{17}$

Nevertheless, the notion of greater PD specificity of pSyn immunohistochemistry of peripheral tissues gains some support from recent studies of peripheral 
sensory and autonomic nerve fibers from skin punch biopsies. While $\alpha$-synuclein staining of autonomic fibers was observed in both patients with PD and controls-although with greater accumulation in $\mathrm{PD}^{26}$ — a recent report found pSyn immunopositivity of dermal nerve fibers only in PD cases, where it was observed in more than $50 \%$, but in none of the controls. ${ }^{23,27}$

There are several possible reasons for the inconsistencies between studies on $\alpha$-synuclein in the ENS. These include differences in applied antibodies and histologic techniques as well as site and depth of biopsies. Since in this study pSyn immunopositivity was only seen in submucosal tissue, but not in the mucosa, appropriate depth of biopsy seems to be critical for the evaluation of pathologic $\alpha$-synuclein in the ENS of patients with PD. In addition, several groups have noted a rostro-caudal gradient with greater density of $\alpha$-synuclein expression in rostral as compared to more caudal portions of the gastrointestinal tract. ${ }^{3,28}$ This suggests that localization of biopsies along the gastrointestinal tract may be another critical factor to be standardized in studies of enteric $\alpha$-synuclein immunohistochemistry. In the current study, however, $\alpha$-synuclein immunoreactivity differences did not appear to depend on the rostro-caudal level of colonic biopsy sites, but the number of cases with multiple biopsies along the rostrocaudal extension of the colon was small.

This is a novel study investigating colonic biopsies of patients with iRBD. This condition is wellestablished as the most specific clinical risk factor for PD identified so far and may indeed represent prodromal PD in up to $80 \% .^{9,11,12}$ Candidate biomarkers for $\mathrm{PD}$ or $\mathrm{PD}$ risk would therefore be expected to show an association with iRBD as has been found for hyposmia, ${ }^{29}$ subtle motor impairment, ${ }^{30}$ impaired color vision, reduced dopamine transporter uptake in the putamen, ${ }^{29,31}$ and hyperechogenicity of the substantia nigra on transcranial ultrasound. ${ }^{32}$ In the present study, positive staining for enteric pSyn was only detected among patients with iRBD and a single patient with PD but in none of our controls. Future studies focusing on expression patterns of phosphorylated $\alpha$-synuclein in peripheral nervous tissue of patients at risk for PD are needed to validate a potential risk of enteric pSyn immunoreactivity as a marker for prodromal PD. Ideally, this should be in the context of prospective cohort studies, where multiple additional markers would be assessed and participants followed longer term to capture the evolution of clinically defined PD. While an ambitious goal, such studies will be critical to define and validate sensitive and specific PD risk markers that could eventually enter screening programs and pave the way to future disease prevention trials.

\section{AUTHOR CONTRIBUTIONS}

F.S. Sprenger: design and conceptualization of the study, statistical analysis and interpretation of the data, drafting and revising the manuscript. N. Stefanova: design and conceptualization of the study, image analysis and interpretation of the data, drafting and revising the manuscript. E. Gelpi: design and conceptualization of the study, image analysis and interpretation of the data, drafting and revising the manuscript. K. Seppi: design and conceptualization of the study, statistical analysis and interpretation of the data, drafting and revising the manuscript. F. Offner: conceptualization of the study, interpretation of the data, revising the manuscript. D. Vilas: interpretation of the data, drafting and revising the manuscript. F. Valldeoriola: interpretation of the data, drafting and revising the manuscript. J. Navarro-Otano: interpretation of the data, drafting and revising the manuscript. I. Aldecoa: interpretation of the data, drafting and revising the manuscript. C. Gaig: interpretation of the data, drafting and revising the manuscript. C. Pont-Sunyer: interpretation of the data, drafting and revising the manuscript. M. Cuatrecasas: interpretation of the data, drafting and revising the manuscript. A. Gines: interpretation of the data, drafting and revising the manuscript. B. Högl: conceptualization of the study, interpretation of the data, revising the manuscript. B. Frauscher: conceptualization of the study, interpretation of the data, revising the manuscript. A. Iranzo: conceptualization of the study, interpretation of the data, drafting and revising the manuscript. G.K. Wenning: conceptualization of the study, interpretation of the data, revising the manuscript. W. Vogel: conceptualization of the study, interpretation of the data, revising the manuscript. E. Tolosa: conceptualization of the study, interpretation of the data, drafting and revising the manuscript. W. Poewe: design and conceptualization of the study, analysis and interpretation of the data, drafting and revising the manuscript.

\section{ACKNOWLEDGMENT}

This study was made possible through the SINBAR (Sleep Innsbruck Barcelona) collaboration.

\section{STUDY FUNDING}

This study was financed in part by grant F4414 of the Austrian Science Fund (FWF) to N.S. and G.K.W. and by a grant/PI13/01455 to F.V. from Proyectos de Investigación en Salud 2013, Instituto de Salud Carlos III, Ministerio de Economía y Competitividad, Gobierno de España.

\section{DISCLOSURE}

The authors report no disclosures relevant to the manuscript. Go to Neurology.org for full disclosures.

Received March 10, 2015. Accepted in final form June 23, 2015.

\section{REFERENCES}

1. den HJW, Bethlem J. The distribution of Lewy bodies in the central and autonomic nervous systems in idiopathic paralysis agitans. J Neurol Neurosurg Psychiatry 1960;23:283-290.

2. Braak H, de Vos RA, Bohl J, Del Tredici K. Gastric alphasynuclein immunoreactive inclusions in Meissner's and Auerbach's plexuses in cases staged for Parkinson's diseaserelated brain pathology. Neurosci Lett 2006;396:67-72.

3. Beach TG, Adler CH, Sue LI, et al. Multi-organ distribution of phosphorylated alpha-synuclein histopathology in subjects with Lewy body disorders. Acta Neuropathol 2010;119:689-702.

4. Pouclet H, Lebouvier T, Coron E, et al. A comparison between rectal and colonic biopsies to detect Lewy pathology in Parkinson's disease. Neurobiol Dis 2012;45:305-309.

5. Lebouvier T, Neunlist M, Bruley des Varannes S, et al. Colonic biopsies to assess the neuropathology of Parkinson's disease and its relationship with symptoms. PLoS One 2010;5:e12728.

6. Shannon KM, Keshavarzian A, Mutlu E, et al. Alpha-synuclein in colonic submucosa in early untreated Parkinson's disease. Mov Disord 2012;27:709-715. 
7. Gold A, Turkalp ZT, Munoz DG. Enteric alpha-synuclein expression is increased in Parkinson's disease but not Alzheimer's disease. Mov Disord 2013;28:237-240.

8. Shannon KM, Keshavarzian A, Dodiya HB, Jakate S, Kordower JH. Is alpha-synuclein in the colon a biomarker for premotor Parkinson's disease? Evidence from 3 cases. Mov Disord 2012;27:716-719.

9. Iranzo A, Molinuevo JL, Santamaria J, et al. Rapid-eyemovement sleep behaviour disorder as an early marker for a neurodegenerative disorder: a descriptive study. Lancet Neurol 2006;5:572-577.

10. Iranzo A, Serradell M, Vilaseca I, et al. Longitudinal assessment of olfactory function in idiopathic REM sleep behavior disorder. Parkinsonism Relat Disord 2013;19:600-604.

11. Schenck CH, Boeve BF, Mahowald MW. Delayed emergence of a parkinsonian disorder or dementia in $81 \%$ of older men initially diagnosed with idiopathic rapid eye movement sleep behavior disorder: a 16-year update on a previously reported series. Sleep Med 2013;14:744-748.

12. Postuma RB, Gagnon JF, Bertrand JA, Genier Marchand D, Montplaisir JY. Parkinson risk in idiopathic REM sleep behavior disorder: preparing for neuroprotective trials. Neurology 2015;84:1104-1113.

13. Hughes AJ, Daniel SE, Kilford L, Lees AJ. Accuracy of clinical diagnosis of idiopathic Parkinson's disease: a clinico-pathological study of 100 cases. J Neurol Neurosurg Psychiatry 1992;55:181-184.

14. Goetz CG, Tilley BC, Shaftman SR, et al. Movement Disorder Society-sponsored revision of the Unified Parkinson's Disease Rating Scale (MDS-UPDRS): scale presentation and clinimetric testing results. Mov Disord 2008;23:2129-2170.

15. Gray MT, Munoz DG, Gray DA, Schlossmacher MG, Woulfe JM. Alpha-synuclein in the appendiceal mucosa of neurologically intact subjects. Mov Disord 2014;29: 991-998.

16. Bottner M, Zorenkov D, Hellwig I, et al. Expression pattern and localization of alpha-synuclein in the human enteric nervous system. Neurobiol Dis 2012;48:474-480.

17. Visanji NP, Marras C, Kern DS, et al. Colonic mucosal $\alpha$-synuclein lacks specificity as a biomarker for Parkinson disease. Neurology 2015;84:609-616.

18. Annerino DM, Arshad S, Taylor GM, Adler $\mathrm{CH}$, Beach TG, Greene JG. Parkinson's disease is not associated with gastrointestinal myenteric ganglion neuron loss. Acta Neuropathol 2012;124:665-680.

19. Fujiwara H, Hasegawa M, Dohmae N, et al. Alpha-synuclein is phosphorylated in synucleinopathy lesions. Nat Cell Biol 2002;4:160-164.
20. Tenreiro S, Eckermann K, Outeiro TF. Protein phosphorylation in neurodegeneration: friend or foe? Front $\mathrm{Mol}$ Neurosci 2014;7:42.

21. Angot E, Steiner JA, Hansen C, Li JY, Brundin P. Are synucleinopathies prion-like disorders? Lancet Neurol 2010;9:1128-1138.

22. Pan-Montojo F, Schwarz M, Winkler C, et al. Environmental toxins trigger $\mathrm{PD}$-like progression via increased alpha-synuclein release from enteric neurons in mice. Sci Rep 2012;2:898.

23. Orimo S, Uchihara T, Nakamura A, et al. Axonal alphasynuclein aggregates herald centripetal degeneration of cardiac sympathetic nerve in Parkinson's disease. Brain 2008; 131:642-650.

24. Doppler K, Ebert S, Uceyler N, et al. Cutaneous neuropathy in Parkinson's disease: a window into brain pathology. Acta Neuropathol 2014;128:99-109.

25. Pouclet $H$, Lebouvier $T$, Coron E, Des Varannes SB, Neunlist M, Derkinderen P. A comparison between colonic submucosa and mucosa to detect Lewy pathology in Parkinson's disease. Neurogastroenterol Motil 2012;24:e202e205. doi: 10.1111/j.1365-2982.2012.01887.x.

26. Wang N, Gibbons CH, Lafo J, Freeman R. Alpha-synuclein in cutaneous autonomic nerves. Neurology 2013;81: 1604-1610.

27. Donadio V, Incensi A, Leta V, et al. Skin nerve alphasynuclein deposits: a biomarker for idiopathic Parkinson disease. Neurology 2014;82:1362-1369.

28. Gelpi E, Navarro-Otano J, Tolosa E, et al. Multiple organ involvement by alpha-synuclein pathology in Lewy body disorders. Mov Disord 2014;29:1010-1018.

29. Postuma RB, Gagnon JF, Vendette M, Desjardins C, Montplaisir JY. Olfaction and color vision identify impending neurodegeneration in rapid eye movement sleep behavior disorder. Ann Neurol 2011;69:811-818.

30. Postuma RB, Lang AE, Gagnon JF, Pelletier A, Montplaisir JY. How does parkinsonism start? Prodromal parkinsonism motor changes in idiopathic REM sleep behaviour disorder. Brain 2012;135:1860-1870.

31. Iranzo A, Lomena F, Stockner H, et al. Decreased striatal dopamine transporter uptake and substantia nigra hyperechogenicity as risk markers of synucleinopathy in patients with idiopathic rapid-eye-movement sleep behaviour disorder: a prospective study [corrected]. Lancet Neurol 2010;9:1070-1077.

32. Iranzo A, Stockner H, Serradell M, et al. Five-year followup of substantia nigra echogenicity in idiopathic REM sleep behavior disorder. Mov Disord 2014;29:1774-1780.

\section{Subspecialty Alerts by E-mail!}

Customize your online journal experience by signing up for e-mail alerts related to your subspecialty or area of interest. Access this free service by visiting Neurology.org/site/subscriptions/etoc.xhtml or click on the "E-mail Alerts" link on the home page. An extensive list of subspecialties, methods, and study design choices will be available for you to choose from—allowing you priority alerts to cutting-edge research in your field! 


\section{Neurology}

\section{Enteric nervous system $\alpha$-synuclein immunoreactivity in idiopathic REM sleep behavior disorder}

Fabienne S. Sprenger, Nadia Stefanova, Ellen Gelpi, et al. Neurology 2015;85;1761-1768 Published Online before print October 16, 2015

DOI 10.1212/WNL.0000000000002126

\section{This information is current as of October 16, 2015}

\section{Updated Information \& Services}

Supplementary Material

\section{References}

\section{Citations}

\section{Subspecialty Collections}

Permissions \& Licensing

\section{Reprints}

including high resolution figures, can be found at: http://n.neurology.org/content/85/20/1761.full

Supplementary material can be found at: http://n.neurology.org/content/suppl/2015/10/16/WNL.0000000000002 126.DC1

This article cites 32 articles, 6 of which you can access for free at: http://n.neurology.org/content/85/20/1761.full\#ref-list-1

This article has been cited by 5 HighWire-hosted articles: http://n.neurology.org/content/85/20/1761.full\#\#otherarticles

This article, along with others on similar topics, appears in the following collection(s):

\section{Parkinson's disease/Parkinsonism}

http://n.neurology.org/cgi/collection/parkinsons_disease_parkinsonism

Information about reproducing this article in parts (figures,tables) or in its entirety can be found online at:

http://www.neurology.org/about/about_the_journal\#permissions

Information about ordering reprints can be found online:

http://n.neurology.org/subscribers/advertise

Neurology ${ }^{\circledR}$ is the official journal of the American Academy of Neurology. Published continuously since 1951, it is now a weekly with 48 issues per year. Copyright () 2015 American Academy of Neurology. All rights reserved. Print ISSN: 0028-3878. Online ISSN: 1526-632X.

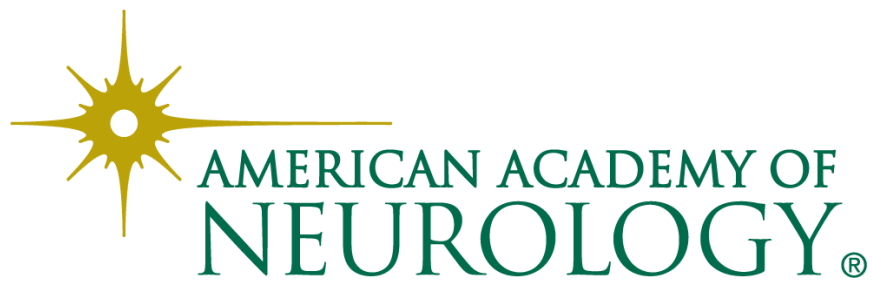

